\title{
Influência de sistemas de preparo na manutenção da palhada e resistência do solo à penetração
}

\author{
Gustavo de Faria Theodoro ${ }^{1}$, Henrique de Oliveira Golin ${ }^{1}$, Rafael Padilha Rezende ${ }^{1}$, Victor \\ Luan Silva de Abreu ${ }^{1}$, Marcio Santos da Silva ${ }^{1}$
}

\begin{abstract}
${ }^{1}$ Universidade Federal de Mato Grosso do Sul, Câmpus de Campo Grande, Campo Grande, Mato Grosso do Sul, Brasil. E-mail: gustavo.theodoro@ufms.br, henriquegolin1@gmail.com, rafinharezende7@gmail.com, viictorabreu@gmail.com, marcio_ms_123@hotmail.com
\end{abstract}

Recebido: 10/11/2017; Aceito: 23/03/2018.

\section{RESUMO}

O preparo do solo favorece o desenvolvimento do sistema radicular e cria condições adequadas para o desenvolvimento das plantas. Todavia, o intenso uso de máquinas e implementos pode afetar a resistência do solo à penetração (RP) e a manutenção da cobertura vegetal morta na superfície do solo. O objetivo deste trabalho foi avaliar a influência dos sistemas de preparo convencional e reduzido sobre a RP e a cobertura vegetal morta em um Latossolo vermelho de textura muito argilosa, em condições de baixa umidade, além de comparar três métodos na estimativa da palhada. A RP foi avaliada por meio do uso de um penetrômetro de impacto modelo IAA/Planalsucar - Stolf na profundidade de 0,0-0,6m e a cobertura vegetal morta por meio da captura e análise de imagens. Houve diferença significativa entre os tratamentos nas profundidades de 0,15-0,20 m, 0,45-0,50 m e 0,50-0,55 m do solo. Ambos os sistemas de preparo fizeram com que o solo apresentasse de baixa a muito alta RP nas profundidades avaliadas. Verificou-se que os métodos para estimar a cobertura morta do solo não diferiram significativamente entre si e que, no momento anterior ao preparo, havia mais de $91 \%$ do solo coberto com palhada. Após as operações mecanizadas, houve maior redução de palha quando foi empregado o sistema de preparo convencional.

Palavras-chave: palha; compactação do solo; grade de discos; arado.

\section{Influence of tillage systems on straw maintenance and soil penetration resistance}

\begin{abstract}
Soil tillage contributes to the development of root system and creates adequate conditions for plants development. However, the intensive use of agricultural machines and implements can affect the mechanical soil resistance to penetration (RP). The objectives of this work were to evaluate the influence of conventional and reduced soil tillage systems on the PR and maintenance of soil cover of a highly clayey texture Red Latosol (Oxisol), besides compare four methods to evaluate the soil cover. An IAA/Planalsucar - Stolf impact penetrometer was used in the depth of $0.0-0.6 \mathrm{~m}$ and the soil cover by capture and analyses of images. There was a significant difference between the treatments in the depths of $0.15-0.20 \mathrm{~m}, 0.45-0.50 \mathrm{~m}$ and $0.50-0.55 \mathrm{~m}$ of the soil and both tillage systems had classes from low to very high RP. It was found that the methods for estimation of soil cover were not significantly different from each other and that, at the time of preparation, more than $91 \%$ of the soil was covered with plant residues. It was observed more straw reduction when the conventional tillage system was used.
\end{abstract}

Key words: straw; soil compaction; disc grades; plow. 


\section{Introdução}

Sistemas de preparo mecanizados do solo são empregados com o objetivo de movimentar suas camadas superficiais e favorecer o desenvolvimento das plantas, porém, o emprego excessivo de máquinas e implementos agrícolas é uma das causas da degradação das propriedades físicas do solo (SILVA et al., 2000; STEFANOSKI et al., 2013).

A resistência do solo à penetração representa as condições de desenvolvimento das raízes das plantas e pode ser medida por meio de equipamentos denominados penetrômetros (STOLF, 1991). Elevados valores de resistência à penetração indicam compactação, influenciam negativamente o crescimento das plantas e estão diretamente relacionados ao uso e manejo do solo. Gomes Júnior et al. (2016) constataram o efeito do manejo por meio da avaliação de solos cultivados com diferentes espécies vegetais e, em ordem decrescente, foi verificada maior resistência à penetração naqueles cultivados com cana-de-açúcar, soja, pinhão-manso e floresta nativa. Monteiro et al. (2017) verificaram o efeito de cinco implementos, empregados individualmente, no preparo de um Argissolo Vermelho-Amarelo Distrófico e verificaram que a resistência do solo à penetração foi menor com o arado de disco ou arado de aiveca; a cobertura vegetal do solo após o preparo se manteve maior com o uso de subsolador e a grade leve não foi eficiente para revolver o solo em preparo primário.

A cobertura morta na superfície do solo proporciona diversos benefícios aos sistemas de produção agropecuários, tais como a proteção dos raios solares e do vento, redução da taxa de evaporação, aumento da infiltração e do armazenamento de água no solo, diminuição de plantas invasoras e aumento da microbiota (HECKLER; SALTON, 2002). Estudos indicam que, além de preservar as propriedades físicas do solo, um manejo que mantenha a cobertura morta aumenta os teores de matéria orgânica, retém formas orgânicas de compostos nitrogenados e aumenta a eficiência de uso de fertilizantes (GUIMARÃES et al., 2013; HUBBARD et al., 2013). Além de atender aos princípios de conservação do solo, a manutenção de resíduos vegetais mortos em sua superfície contribui com a disponibilização de nutrientes para os cultivos em sucessão (ROSOLEM et al., 2003).

A estimativa da cobertura morta é empregada para classificar sistemas conservacionistas de preparo periódico do solo (ZHENG et al., 2014) e os principais métodos para este fim são o fotográfico e o de transeção linear, com diversas variações (SLONEKER; MOLDENHAUER, 1977; RICHARDS et al., 1984; BOER et al., 2008; ZHENG et al., 2014). No Rio Grande do Sul, Alves et al. (1998) estimaram o porcentual do solo ocupado por restos vegetais oriundos da colheita da soja por meio dos métodos de transeção linear de Sloneker e Moldenhauer (1977) e fotográfico (JORGE; SILVA, 2009). Verificou-se que em áreas sob gradagem ou escarificação do solo os índices de cobertura obtidos pelo método fotográfico foram superiores aos da transeção linear, enquanto que na área sem preparo do solo houve similaridade entre os resultados dos dois métodos.

No Centro-Oeste brasileiro, em anos com atraso das chuvas após longo período de estiagem no inverno, muitos agropecuaristas iniciam as operações de preparo em condições de baixa umidade do solo. Estes produtores, de uma forma geral, possuem pouca diversidade de implementos agrícolas, restringindo-se a um arado, uma grade niveladora e uma grade aradora. $\mathrm{O}$ objetivo deste trabalho foi comparar métodos para estimar a cobertura vegetal morta sobre o solo e o efeito de sistemas de preparo com poucos implementos, em condições de baixa umidade, na manutenção da palhada e a resistência do solo à penetração.

\section{Material e Métodos}

\subsection{Comparação de métodos para estimar a palhada sobre o solo}

O trabalho foi realizado em área experimental da Faculdade de Medicina Veterinária e ZootecniaFAMEZ da Universidade Federal de Mato Grosso do Sul, localizado na cidade Campo Grande (20²6'34" S 543' $47^{\prime \prime}$ W) a $532 \mathrm{~m}$ de altitude. O solo foi classificado como Latossolo Vermelho (EMBRAPA, 2013), com textura argilosa (590 $\mathrm{g} \mathrm{kg}^{-1}$ de argila, $110 \mathrm{~g}$ $\mathrm{kg}^{-1}$ de areia e $300 \mathrm{~g} \mathrm{~kg}^{-1}$ de silte) e o clima da região identificado como Aw - clima tropical, com inverno seco (KOTTEK et al., 2006).

O solo estava ocupado com capim Brachiaria e recebeu uma operação tratorizada com grade de discos lisos de 28 polegadas. Quando os restos culturais estavam secos, com coloração parda, houve o início das avaliações.

Foram empregados quatro tratamentos, representados por métodos de transeção linear e fotográfico para se estimar a cobertura vegetal morta no solo. Os três métodos de transeção linear empregados se basearam no uso de um fio (barbante) com pontos equidistantes, estendido sobre o solo para se registrar o número de vezes em que estes pontos ficaram sobrepostos às unidades de palha . Posteriormente, calculou-se o porcentual de pontos com interceptação de cobertura vegetal morta. A diferença entre os métodos se baseou no tamanho do fio e na distância entre os pontos, conforme segue: a) fio com 11,54 m de extensão e 50 pontos espaçados de 0,23 m (SLONEKER; MOLDENHAUER, 1977); b) quadrado de arame de $1 \mathrm{~m}^{2}$, com um fio disposto em diagonal e 13 pontos 
espaçados de 0,05 m (BOER et al., 2008); c) fio com 15 $\mathrm{m}$ de extensão e 100 pontos espaçados de $0,15 \mathrm{~m}$ (RICHARDS et al., 1984).

O método fotográfico consistiu na captura de imagens de uma área de $1,5 \mathrm{~m}^{2}$ a $1,6 \mathrm{~m}$ de altura, formando um ângulo de $90^{\circ} \mathrm{com}$ a superfície do solo por meio de um dispositivo provido de câmera com 13 MP . Posteriormente, as imagens foram analisadas no programa SisCob 1.0 (JORGE; SILVA, 2009), por via de rede neural e duas cores pré-definidas para palha e solo. Foram coletadas cinco imagens para a composição da média de cada parcela experimental medindo $60 \mathrm{~m}^{2}$.

O delineamento experimental utilizado foi de blocos casualizados, com quatro tratamentos e cinco repetições. Com base na normalidade das variâncias, os dados foram transformados em $\sqrt{\mathrm{x}}$ e submetidos à análise de variância. As médias dos tratamentos foram comparadas pelo teste de Tukey, a 5\% de probabilidade empregando-se o software Assistat (SILVA; AZEVEDO, 2016).

\subsection{Influência de sistemas de preparo sobre a} resistência à penetração do solo e a manutenção da palhada sobre o solo

$\mathrm{O}$ experimento foi conduzido na fazenda escola da Universidade Federal do Mato Grosso do Sul, da Faculdade de Medicina Veterinária e Zootecnia (20²6'50.6"S 5450'34.0"W), no município de Terenos a 407 metros de altitude. O solo foi classificado como Latossolo Vermelho, com textura muito argilosa (660 g $\mathrm{kg}^{-1}$ de argila, $210 \mathrm{~g} \mathrm{~kg}^{-1}$ de areia, $130 \mathrm{~g} \mathrm{~kg}^{-1}$ de silte) e o clima da região identificado como Aw - Clima tropical, com inverno seco. O solo estava ocupado por intensa vegetação espontânea e capim braquiária que foram dessecados por meio de aplicação de glifosato (3 L.ha ${ }^{-1}$ ) na primeira quinzena de abril de 2017.

Empregando-se um trator de 90 cavalos e $3.580 \mathrm{~kg}$ foi realizada uma operação com grade aradora de 14 discos recortados, com 36 polegadas em área total para criar condições favoráveis ao preparo primário do solo. Posteriormente, foram utilizados dois sistemas, identificados como reduzido e convencional, considerados como tratamentos. O preparo reduzido consistiu em duas gradagens, uma com grade aradora e outra com grade niveladora "off set", com 42 discos lisos de 28 polegadas. O preparo convencional consistiu em uma operação com arado com quatro discos lisos de 42 polegadas seguida de quatro com a grade niveladora. Logo após o preparo do solo, coletaram-se amostras nas profundidades de 0,0-0,2 m, 0,2-0,4 m e 0,4-0,6 m para se determinar a umidade gravimétrica do solo de acordo com o método descrito por Embrapa (1997).

A resistência do solo à penetração $(\mathrm{RP})$ foi estimada empregando-se o penetrômetro de impacto modelo IAA/Planalsucar-Stolf nas mesmas profundidades em que se efetuou a coleta do solo para se estimar a umidade gravimétrica, conforme Stolf et al. (2012). Os dados foram obtidos em impactos por centímetros e, em seguida, transformados para a unidade de pressão $\mathrm{MPa}$ por meio da fórmula descrita por Stolf (1991). A interpretação dos resultados foi feita conforme a classificação da resistência do solo empregada pelo United States Department of Agriculture, citada por Gomes Júnior. et al. (2016), conforme segue: extremamente baixa $<0,01 \mathrm{MPa}$; muito baixa $0,01-0,1$ Mpa; baixa 0,1 - 1,0 Mpa; moderada 1,0 - 2,0 Mpa; alta 2,0 - 4,0 Mpa; muito alta 4,0 - 8,0 Mpa e extremamente alta $>8 \mathrm{Mpa}$.

A avaliação foi realizada por meio da análise de imagens, que foram capturadas e analisadas de acordo com o método descrito no ítem 2.1. O delineamento experimental empregado foi de blocos ao acaso, com 10 repetições por tratamento em esquema fatorial $2 \times 2$ a saber: dois sistemas de preparo do solo (convencional e reduzido) e duas épocas avaliação (antes e depois das operações). Foram empregadas 10 repetições por tratamento; cada parcela experimental possuindo 9 (análise da RP) e $30 \mathrm{~m}^{2}$ (estimativa da cobertura vegetal morta) e realizadas três leituras com o penetrômetro de impacto nas profundidades analisadas e três fotografias em pontos diferentes da superfície. O valor de cada repetição foi obtido por meio da média aritmética. Os efeitos dos tratamentos em cada profundidade foram analisados separadamente e, quando necessário, as médias foram transformadas em $V_{\mathrm{x}}$ e as médias comparadas pelo teste de Tukey, a 5\% de probabilidade.

\section{Resultados e Discussão}

Na profundidade de 0,05-0,1 m, ambos os preparos proporcionaram baixa RP do solo. $\mathrm{Na}$ profundidade média de $0,125 \mathrm{~m}$, o solo preparado de forma convencional apresentou moderada RP (1,59 Mpa), enquanto que no solo sob preparo reduzido houve alta RP (2,76 Mpa). A RP do solo na profundidade de 0,15$0,20 \mathrm{~m}$ foi identificada como muito alta tanto na área preparada de forma convencional $(6,0 \mathrm{MPa})$ quanto na reduzida (4,1 Mpa).

Apesar do preparo convencional estar relacionado a um número relativamente elevado de operações mecanizadas que reduzem o tamanho dos torrões/agregados do solo surgidos após a aração do solo, apenas houve diferença significativa na profundidade média de 17,5 $\mathrm{cm}$ (camada de 0,15-0,20 $\mathrm{m})$. As várias operações com a grade niveladora foram necessárias em função da baixa umidade do solo em todos os estratos de profundidade. $\mathrm{Na}$ profundidade abaixo dos 0,2 m, observou-se RP do solo alta e muito alta, havendo diferença significativa entre os dois tratamentos na profundidade média de 0,475 e 0,525 m. 
Esta diferença provavelmente não foi causada pelos tratamentos empregados e indica a necessidade do preparo do solo em profundidade. $\mathrm{O}$ menor valor de RP foi observado nas camadas superficiais do solo, enquanto que o maior valor ocorreu no preparo convencional e na profundidade média de $0,275 \mathrm{~m}(7,1$ Mpa), indicando a necessidade de monitoramento e o preparo em camadas sub superficiais. A RP em profundidades maiores foi reduzida pois provavelmente houve menor influência dos discos de corte dos implementos e do rodado do trator empregados.

Diversos estudos mostram a influência da mecanização na RP dos solos (SILVA et al., 2000; GOMES et al., 2016). Esses resultados corroboram com RALISCH et al. (2008), que verificaram, sob plantio convencional, menor resistência do solo à penetração na profundidade $0-0,10 \mathrm{~m}$, enquanto que com dois anos de plantio direto houve maior resistência à penetração até 0,40 m de profundidade. Por outro lado, ao avaliar o preparo convencional, cultivo mínimo com vibro escarificador, cultivo mínimo com grade leve e plantio direto em um Argisolo Vermelho-Amarelo com umidade média em condições de campo de $0,26 \mathrm{~kg} \mathrm{~kg}^{-1}$, Fernandes et al. (2008) não constaram diferença significativa na RP entre os tratamentos.

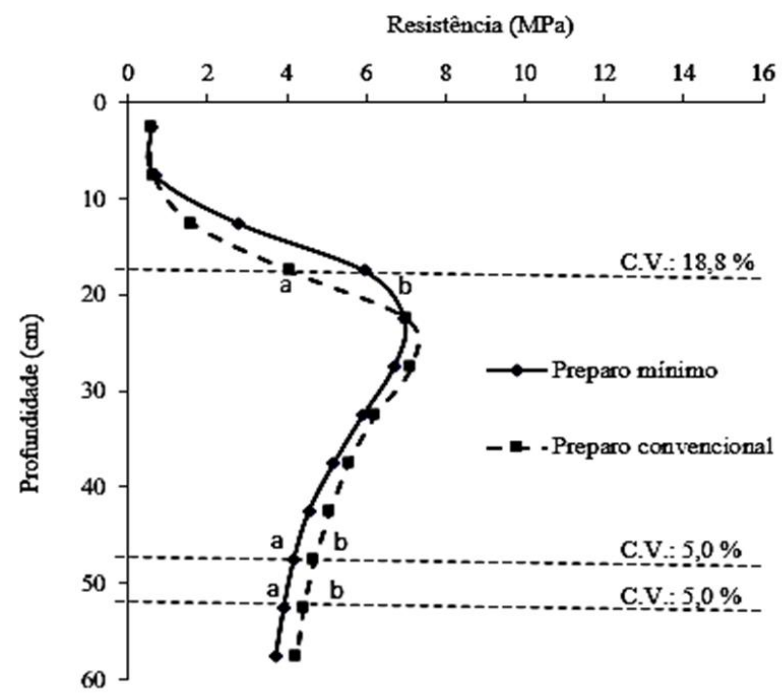

Figura 1. Influência do preparo reduzido e convencional na resistência à penetração (MPa) de um Latossolo Vermelho de textura muito argilosa sob vegetação espontânea. Médias na linha seguidas de mesma letra não diferem pelo teste Tukey a 5\% de significância. C.V.(\%): coeficiente de variação. Terenos, MS.

O presente trabalho foi desenvolvido em condições climáticas encontradas pelos produtores rurais da região central do estado de Mato Grosso do Sul, época em que ocorre atraso nas chuvas após uma longa estiagem no inverno. Durante o preparo do solo, a umidade gravimétrica nas camadas do solo foi relativamente baixa $\left(0,21 \quad \mathrm{~kg} \quad \mathrm{~kg}^{-1}\right)$. Apesar da discussão se fundamentar em parâmetros relacionados às dificuldades proporcionadas ao desenvolvimento do sistema radicular das plantas, esta deve ser feita de forma criteriosa pois a semeadura ocorre apenas após eventos de precipitação pluvial que, dependendo de sua intensidade, podem reduzir significativamente os valores de RP do solo (OLIVEIRA et al., 2007). Desta forma, é possível concluir que ambos os sistemas de preparo influenciaram o solo de forma muito semelhante no momento das operações, apesar da variação nos valores de RP observada. Uma elevada amplitude na RP também foi constatada por Freddi et al. (2017), com valores de até 9,37 MPa em um Latossolo Vermelho Amarelo após 11 anos de sucessão de soja e milho, em semeadura direta e valor médio de umidade gravimétrica de $0,26 \mathrm{~kg} \mathrm{~kg}^{-1}$.

Verificou-se que os métodos avaliados para estimar a cobertura vegetal morta do solo não diferiram significativamente entre si (Tabela 1). Em média e conforme os métodos avaliados, 49,7 \% da superfície do solo esteve ocupado com resíduos vegetais mortos. Estes resultados corroboram com Laamrani et al. (2017), que verificaram forte correlação dos resultados obtidos pelo método de transeção linear (RICHARDS et al. 1984) e de análise digital de imagens em campos de produção em Ontário, Canadá.

A comparação de métodos de transeção linear realizada neste trabalho foi inédita e mostrou que todos foram significativamente semelhantes ao estimar a cobertura morta do solo. Sugere-se que a escolha do método deva ser em função da comodidade, mão de obra treinada, tempo disponível e/ou disponibilidade de dispositivo fotográfico. O método fotográfico pela captura das imagens para análise foi mais ágil do que o método de transeção linear, sendo uma opção atrativa e semelhantemente confiável. Por outro lado, há desvantagens elencadas por Alves et al (1998) e que foram confirmadas durante a condução deste trabalho como: maior custo operacional, impossibilidade de conhecimento dos dados ainda no campo e risco de perda dos dados por problemas na captura das fotos ou em seu armazenamento.

Os sistemas de preparo exercem grande influência na preservação da cobertura vegetal morta do solo (Tabela 2). Verificou-se que, antes do preparo, a área do solo ocupada com cobertura vegetal foi elevada e significativamente semelhante. Após o preparo convencional do solo, houve redução de $68 \%$ da cobertura morta, resultado do revolvimento da camada superficial do solo pelo arado e as subsequentes operações com grade niveladora para a desestruturação de torrões incorporados à palhada existente na superfície do solo. O preparo reduzido proporcionou a manutenção de $71,1 \%$ da cobertura morta no solo, uma vez que apenas o uso das grades aradora e niveladora foi suficiente para deixar o solo apto para a semeadura. 
Tabela 1. Comparação de métodos para estimar a cobertura vegetal morta do solo. Campo Grande, MS

\begin{tabular}{lll}
\multicolumn{1}{c}{ Tratamentos } & \multicolumn{1}{c}{ Descrição } & Cobertura vegetal morta \\
$(\%)$
\end{tabular}

* Não significativo a 5\% de probabilidade. Dados transformados em $\sqrt{\mathrm{x}}$.

Os resultados obtidos neste trabalho mostraram que o preparo do solo de forma convencional não contribuiu com a pouca cobertura do solo com restos vegetais mortos, o que o torna vulnerável a processos erosivos (LAL, 1984). Já o preparo reduzido proporcionou uma elevada quantidade de cobertura vegetal morta remanescente $(71,1 \%)$, uma vez que se considera que o sistema de plantio direto deva ter pelo menos $50 \%$ da superfície do solo coberta com resíduos vegetais (ALVARENGA et al., 2001).

Entende-se como benéfico ao meio ambiente e vantajoso ao produtor rural a priorização de sistemas de preparo de solo que promovam a manutenção da maior quantidade de palha em sua superfície, pois promovem menos perda de solo por processos erosivos. Cassol e Lima (2003) avaliaram a erosão hídrica em entressulcos em função de sistemas de manejo de um Argissolo Vermelho, e concluíram que as taxas de perdas de solo por erosão foram reduzidas em torno de $90 \%$ pelo sistema sem preparo do solo em relação ao solo descoberto e ao preparo convencional com incorporação de resíduos. No solo descoberto, sem palhada, houve uma maior exposição da superfície do solo ao impacto das gotas da chuva e a formação de selamento superficial.

Tabela 2. Efeito dos sistemas de preparo mecanizado na cobertura vegetal (\%) morta sobre o solo. Terenos, MS.

\begin{tabular}{lcc}
\hline \multicolumn{1}{c}{ Época de } & \multicolumn{2}{c}{ Sistema de preparo do solo } \\
\cline { 2 - 3 } \multicolumn{1}{c}{ avaliação } & Convencional & Reduzido \\
\hline Antes & $91,7 \mathrm{aA}$ & $93,7 \mathrm{aA}$ \\
Depois & $23,7 \mathrm{bB}$ & $71,1 \mathrm{bA}$ \\
\hline C.V. $(\%)$ & & 16,1
\end{tabular}

Médias na linha seguidas de mesma letra maiúscula não diferem pelo teste Tukey a 5\% de significância; médias na coluna seguidas de mesma letra minúscula não diferem pelo teste Tukey a 5\% de significância.

\section{Conclusões}

Os dois sistemas de preparo influenciaram a resistência à penetração do solo nas diferentes profundidades avaliadas, porém, nas camadas superficiais proporcionaram os mesmos efeitos na resistência à penetração.
Não houve diferença entre os métodos avaliados para estimar a cobertura morta do solo.

O solo preparado de forma convencional apresentou a menor área coberta com resíduos vegetais em sua superfície.

\section{Referências Bibliográficas}

ALVARENGA, R. C.; LARA CABEZAS, W. A.; CRUZ, J. C.; SANTANA, D. Plantas de cobertura de solo para sistema plantio direto. Informe Agropecuário, Belo Horizonte-MG, v. 22 , n. 208 , p. 25-36, 2001.

ALVES, A. G. C.; COGO, N. P.; LEVIEN, R. Comparação entre os métodos da transeção linear e fotográfico na avaliação de cobertura vegetal morta, sob dois métodos de preparo, após a colheita da soja. Revista Brasileira de Ciência do Solo, Viçosa-MG, v. 22, n. 3, p. 491-496, 1998.

BOER, C. A A.; ASSIS, R. L.; SILVA, G. P.; BRAZ, A. J. B. P.; BARROSO, A. L. L.; CARGNELUTTI FILHO, A.; PIRES, F. R. Biomassa, decomposição e cobertura do solo ocasionada por resíduos culturais de três espécies vegetais na região Centro-Oeste do Brasil. Revista Brasileira de Ciência do Solo, Viçosa-MG, v. 32, p. 843-851, 2008.

CASSOL, E.A.; LIMA, V.S. Erosão em entressulcos sob diferentes tipos de preparo e manejo do solo. Pesquisa Agropecuária Brasileira, Brasília-DF, v. 38, n. 1, p. 117-124, 2003.

EMBRAPA. EMPRESA BRASILEIRA DE PESQUISA AGROPECUÁRIA Manual de métodos de análise de solos. 2. ed., Rio de Janeiro-RJ: Embrapa Solos, 1997. 212 p.

EMBRAPA. EMPRESA BRASILEIRA DE PESQUISA AGROPECUÁRIA. Sistema brasileiro de classificação de solos. Brasília-DF: Embrapa Solos, 2013. 353p.

FERNANDES, H.C.; SILVEIRA, J.C.M.; RINALDI, P.C.N. Avaliação do custo energético de diferentes operações agrícolas mecanizadas. Ciência e Agrotecnologia, LavrasMG, v. 32, n. 5, p. 1582-1587, 2008.

FREDDI, O. S.; TAVANTI, R. F. R.; SOARES, M. B.; ALMEIDA, F. T.; PERES, F. S. C. Physical-chemical quality of a Latossol under direct seeding and soybean-corn succession in the cerrado-amazonian ecotone. Revista Caatinga, Mossoró-RN, v. 30, n. 4, p. 991-1000, 2017. 
GOMES JUNIOR, D. G.; STOLF, R.; PERES, J. G.; PINTO, V. M.; REICHARDT, K. Soil physical quality of Brazilian crop management systems evaluated with aid of penetrometer. Journal of Agricultural Science, Ontario, v. 8, n. 6, p. 120$128,2016$.

GUIMARÃES, D. V.; GONZAGA, M. I. S.; SILVA, T. O.; SILVA, T. L.; DIAS, N. S.; MATIAS, M. I. S. Soil organic matter pools and carbon fractions in soil under different land uses. Soil and Tillage Research, Amsterdam, v. 126, n. 1, p. 177-182, 2013.

HECKLER, J. C.; SALTON, J. C. Palha: fundamento do sistema plantio direto. Dourados-MS: Embrapa Agropecuária Oeste, 2002. 26 p.

HUBBARD, R. K.; STRICKLAND, T. C.; PHATAK, S. Effects of cover crop systems on soil physical properties and carbon/nitrogen relationships in the coastal plain of southeastern USA. Soil and Tillage Research, Amsterdam, v. 126, p. 276-283, 2013.

KOTTEK, M.; GRIESER, J.; BECK, C.; RUDOLF, B.; RUBEL, F. World map of the Köppen-Geiger climate classification updated. Meteorologische Zeitschrift, v. 15, n. 3, p. 259-263, 2006.

JORGE, L. A. C.; SILVA, D. J. C. B. SisCob: manual de utilização. São Carlos-SP: Embrapa Instrumentação Agropecuária, 2009. 18 p.

LAAMRANI, A.; JOOSSE, P.; FEISTHAUER, N. Determining the number of measurements required to estimate crop residue cover by different methods. Journal of Soil and Water Conservation, Ankeny, v. 72, n. 5, p. 471-479, 2017.

LAL, R. Mechanized tillage systems effects on soil erosion from an Alfisol in watersheds cropped to maize. Soil and Tillage Research, Amsterdam, v. 4, p. 349-360, 1984.

MONTEIRO, M. A. C.; ZOZ, A.; LIMEDE, A. C.; OLIVEIRA, C. E. S.; ZOZ, T. Efeito do preparo do solo com diferentes implementos sobre a resistência do solo à penetração. Revista de Agricultura Neotropical, Cassilândia-MS, v. 4, n. 2, p. 63-68, 2017.

OLIVEIRA, G. C.; SEVERIANO, E. C.; MELlO, C. R. Dinâmica da resistência à penetração de um Latossolo Vermelho da Microrregião de Goiânia, GO. Revista Brasileira de Engenharia Agrícola e Ambiental, Campina Grande-PB, v. 11, n. 3, p. 265-270, 2007.

RALISCH，R.; MIRANDA，T. M.; OKUMURA，R. S.; BARBOSA, G. M. C.; GUIMARÃES, M. F.; SCOPEL, E.; BALBINO, L. C. Resistência à penetração de um Latossolo Vermelho Amarelo do Cerrado sob diferentes sistemas de manejo. Revista Brasileira de Engenharia Agrícola e Ambiental, Campina Grande-PB, v. 12, n. 4, p. 381-384, 2008.
RICHARDS, B. K., WALTER, M. F.; MUCK, R. E. Variation in line transect measurements of crop residue cover. Journal of Soil and Water Conservation, Ankeny, v. 39, n. 1, p. 60$61,1984$.

ROSOlEM, C. A.; CAlONEGO, J. C.; FOLONI, J. S. S. Lixiviação de potássio da palha de espécies de cobertura de solo de acordo com a quantidade de chuva aplicada. Revista Brasileira de Ciência do Solo, Viçosa-MG, v. 27, p. 355-362, 2003.

SILVA, F. A. S.; AZEVEDO C. A. V. The Assistat Software version 7.7 and its use in the analysis of experimental data. African Journal of Agriculture Research, Joanesburgo, v. 11, n. 39, p. 3733-3740, 2016.

SILVA, V. R.; REINERT, D. J.; REICHERT, J. M. Resistência mecânica do solo à penetração influenciada pelo tráfego de uma colhedora em dois sistemas de manejo do solo. Ciência Rural, Santa Maria-RS, v. 30, n. 5, p. 795-801, 2000.

SLONEKER, L. L.; MOLDENHAUER, W. C. Measuring the amounts of crop residue remaining after tillage. Journal of Soil and Water Conservation, Ankeny, v. 32, p. 231-236, 1977.

STEFANOSKI, D. C.; SANTOS, G. G.; MARCHÃO, R. L.; PETTER, F. A.; PACHECO, L. P. Uso e manejo do solo e seus impactos sobre a qualidade física. Revista Brasileira de Engenharia Agrícola e Ambiental, Campina Grande-PB, v. 17, n. 12, p. 1301-1309, 2013.

STOLF, R. Teoria e teste experimental de fórmulas de transformação dos dados de penetrômetro de impacto em resistência do solo. Revista Brasileira de Ciência do Solo, Viçosa-MG, v. 15, p. 229-235, 1991.

STOLF, R., MURAKAMI, J. H., MANIERO, M. A., SILVA, L. C. F.; SOARES, M. R. Incorporação de régua para medida de profundidade no projeto do penetrômetro de impacto Stolf. Revista Brasileira de Ciência do Solo, Viçosa-MG, v. 36, n. 5, p. 1476-1482, 2012.

ZHENG, B.; CAMPBELL, J. B.; SERBIN, G.; GALBRAITH, J. M. Remote sensing of crop residue and tillage practices: present capabilities and future prospects. Soil and Tillage Research, Amsterdam, v. 138, p. 26-34, 2014. 